ORIGINAL ARTICLE

\title{
Associations of anthropometric markers with serum metabolites using a targeted metabolomics approach: results of the EPIC-potsdam study
}

\author{
U Bachlechner ${ }^{1}$, A Floegel ${ }^{1}$, A Steffen $^{1}$, C Prehn $^{2}$, J Adamski ${ }^{2,3,4}$, T Pischon $^{5}$ and H Boeing ${ }^{1}$
}

\begin{abstract}
BACKGROUND/OBJECTIVES: The metabolic consequences of type of body shape need further exploration. Whereas accumulation of body mass in the abdominal area is a well-established metabolic risk factor, accumulation in the gluteofemoral area is controversially debated. We evaluated the associations of anthropometric markers of overall body mass and body shape with 127 serum metabolites within a sub-sample of the European Prospective Investigation into Cancer and Nutrition (EPIC)-Potsdam cohort. SUBJECTS/METHODS: The cross-sectional analysis was conducted in 2270 participants, randomly drawn from the EPIC-Potsdam cohort. Metabolites were measured by targeted metabolomics. To select metabolites related with both waist circumference (WC) (abdominal subcutaneous and visceral fat) and hip circumference (HC) (gluteofemoral fat, muscles and bone structure) correlations ( $r$ ) with body mass index (BMI) as aggregating marker of body mass (lean and fat mass) were calculated. Relations with body shape were assessed by median metabolite concentrations across tertiles of WC and HC, mutually adjusted to each other.

RESULTS: Correlations revealed 23 metabolites related to BMI $(r \geqslant 10.20 \mathrm{I})$. Metabolites showing relations with BMI were showing similar relations with $\mathrm{HC}$ adjusted WC $\left(\mathrm{WC}_{\mathrm{HC}}\right)$. In contrast, relations with WC adjusted $\mathrm{HC}\left(\mathrm{HC}_{\mathrm{WC}}\right)$ were less concordant with relations of $\mathrm{BMI}$ and $\mathrm{WC}_{\mathrm{HC}}$. In both sexes, metabolites with concordant relations regarding $\mathrm{WC}_{\mathrm{HC}}$ and $\mathrm{HC}_{\mathrm{WC}}$ included tyrosine, diacylphosphatidylcholine C38:3, C38:4, lyso-phosphatidylcholine C18:1, C18:2 and sphingomyelin C18:1; metabolites with opposite relations included isoleucine, diacyl-phosphatidylcholine C42:0, acyl-alkyl-phosphatidylcholine C34:3, C42:4, C42:5, C44:4 and C44:6. Metabolites specifically related to $\mathrm{HC}_{\text {WC }}$ included acyl-alkyl-phosphatidylcholine C34:2, C36:2, C38:2 and C40:4, and were solely observed in men. Other metabolites were related to $\mathrm{WC}_{\mathrm{HC}}$ only.

CONCLUSIONS: The study revealed specific metabolic profiles for $\mathrm{HC}_{\mathrm{WC}}$ as marker of gluteofemoral body mass differing from those for $\mathrm{BMI}$ and $\mathrm{WC}_{\mathrm{HC}}$ as markers of overall body mass and abdominal fat, respectively. Thus, the study suggests that gluteofemoral mass may have less-adverse metabolic implications than abdominal fat.
\end{abstract}

Nutrition \& Diabetes (2016) 6, e215; doi:10.1038/nutd.2016.23; published online 27 June 2016

\section{INTRODUCTION}

Overweight and obesity are related to metabolic disorders (for example, impaired fasting glucose, impaired glucose tolerance, hypertriglyceridemia, type-2-diabetes). ${ }^{1}$ A major determinant of weight-associated metabolic disorders is the type of body shape. The commonly referred 'apple' shape, characterized by predominating abdominal fat accumulation, is supposed to pose greater health risks than the 'pear' shape, characterized by predominating gluteofemoral fat accumulation. ${ }^{2}$ Health risks related to abdominal fat are mainly driven by the visceral fat compartment, whose expansion is a well-established metabolic risk factor. ${ }^{3,4}$ Expansion of subcutaneous fat, such as gluteofemoral fat, presumably does not have metabolically adverse effects, but is supposed to act protective. . $^{5}$

Overweight and obesity are classified by body mass index (BMI), a simple population level measure, which is defined as one's weight in kilograms divided by the square of one's height in meters. Consequently, BMI is an aggregating marker of body mass covering lean mass (mainly including muscles, bones and water) and fat mass. Using BMI to classify excess body fat is based on the assumption that at a given height, higher weight is associated with increased fatness. ${ }^{7}$ To assess body shape, circumferences such as waist circumference (WC) and hip circumference (HC) are commonly used as surrogate measures. Whereas WC comprises mainly abdominal subcutaneous and visceral fat, HC comprises gluteofemoral fat, muscles and bone structure (pelvic width). Furthermore, it must be borne in mind that anthropometric measures are generally affected by an individual's body frame. Even though circumferences can only provide rough estimates of one's true body composition, WC is supposed to be the most effective single measure for visceral fat. ${ }^{8-10} \mathrm{HC}$ generally reflects gluteofemoral body mass, but it is not able to differentiate between parts attributed to fat, muscles or bone structure. Moreover, parts may clearly differ between men and women. However, in both sexes HC highly correlates with subcutaneous fat. ${ }^{10}$

Excess weight affects the whole body and apparently involves metabolic changes. ${ }^{11-14}$ The metabolomic approach has already

\footnotetext{
${ }^{1}$ Department of Epidemiology, German Institute of Human Nutrition Potsdam-Rehbruecke, Nuthetal, Germany; ${ }^{2}$ Institute of Experimental Genetics, Helmholtz Center Munich, German Research Center for Environmental Health, Neuherberg, Germany; ${ }^{3}$ German Center for Diabetes Research, Neuherberg, Germany; ${ }^{4}$ Institute of Experimental Genetics, Technical University of Munich, Freising-Weihenstephan, Germany and ${ }^{5}$ Molecular Epidemiology Group, Max Delbrück Center for Molecular Medicine (MDC), Berlin-Buch, Germany. Correspondence: U Bachlechner, Department of Epidemiology, German Institute of Human Nutrition Potsdam-Rehbruecke, Arthur-Scheunert-Allee 114-116, Nuthetal 14558, Germany.
} 
been used to identify metabolites involved in overweight and obesity ${ }^{11,15-19}$ and weight-associated metabolic disorders, for example, pre-diabetes (that is, impaired fasting glucose and/or impaired glucose tolerance) ${ }^{12,20-23}$ and clinically manifest type-2diabetes. $^{24-26}$ The metabolic state particularly related with body shape is not fully understood. It is assumed that the accumulation of visceral fat has an important etiological role for weightassociated metabolic disorders, ${ }^{27,28}$ whereas the accumulation of gluteofemoral body mass was found to be associated with more favorable metabolic profiles, when abdominal fat was taken into account. $^{29,30}$ In the present study, we aimed to gain a broader understanding of the metabolic state related to body mass in general and body shape in particular. Therefore, we evaluated the associations of anthropometric markers of overall body mass and body shape with 127 targeted metabolites in chronic disease-free individuals within a sub-sample of the European Prospective Investigation into Cancer and Nutrition (EPIC)-Potsdam cohort.

\section{SUBJECTS AND METHODS}

\section{Ethics statement}

The study was approved by the ethics committee of the Medical Association of the State of Brandenburg. A written informed consent from all participants was obtained.

\section{Study population}

The EPIC-Potsdam cohort is part of the ongoing multicenter EPIC study and comprises 27548 participants from the general population of Potsdam and surroundings, recruited between 1994 and 1998. ${ }^{31}$

The present analysis was conducted in a randomly drawn sub-sample $(n=2500)$ from the EPIC-Potsdam study population with blood samples ( $n=26448$ ). Participants, mainly aged $35-65$ years, filled out sociodemographic and lifestyle questionnaires, a validated food-frequency questionnaire and completed an interview on medical history. Participants additionally had anthropometric measures and a blood sample taken by trained staff. ${ }^{32,33}$ In brief, $30 \mathrm{ml}$ of blood were collected and immediately processed according to a strict protocol. ${ }^{34}$ The blood was fractionated into serum, plasma, buffy coat and erythrocytes, aliquoted into straws of $0.5 \mathrm{ml}$, and stored in liquid nitrogen tanks at $-196^{\circ} \mathrm{C}$ until analysis.

In this cross-sectional study we excluded participants with missing anthropometric measurements, missing blood samples or biomarker measurement, missing covariate information and participants with prevalent chronic diseases. Thus, the analytical sample included 2270 individuals.

\section{Measurement of anthropometry}

Anthropometry was measured according to standardized procedures by trained staff as described earlier. ${ }^{35}$ Weight was obtained without shoes in light clothing to the nearest of $0.1 \mathrm{~kg}$. Height and circumferences were measured to the nearest of $0.1 \mathrm{~cm}$. WC was taken midway between the iliac crest and the lower ribs. $\mathrm{HC}$ was measured over the buttocks. BMI was calculated as weight in kilograms divided by the square of height in meters.

\section{Measurement of serum metabolites}

Metabolomic measurements were performed in the Genome Analysis Center at the Helmholtz Center Munich by electrospray ionization-tandem mass spectrometry using the AbsoluteIDQ p150 kit (BIOCRATES Life Sciences AG, Innsbruck, Austria). Sample preparation has been described in detail previously. ${ }^{36,37}$ In brief, $10 \mu$ lof serum were pipetted onto a filter in a 96-well sandwich plate, containing stable isotope-labeled internal standards. Amino acids (AA) were derivated with $5 \%$ phenylisothiocyanate reagent. After extraction of metabolites and internal standards with $5 \mathrm{~mm}$ ammonium acetate in methanol, the solution was centrifuged through a filter membrane and diluted with mass spectrometry running solvent. Final extracts were analyzed and 163 metabolites quantified simultaneously, including free carnitine, 40 acylcarnitines, 14 AA (13 proteinogenic +ornithine), one hexose (sum of six carbon monosaccharides without distinction of isomers), 92 glycerophospholipids (15 lyso-phosphatidylcholines (lysoPC), 77 diacyl (aa)- and acyl-alkyl (ae)- phosphatidylcholines
$(\mathrm{PC})$ ) and 15 sphingomyelins (SM). Internal standards were used to calculate metabolite concentrations. Fatty-acid side chains were abbreviated Cx:y where $x$ represented the number of carbon atoms and $y$ the number of double bonds. The acylcarnitines were derivates of carnitine (CO) with one fatty-acid bond (CX:y). The prefix 'lyso' indicated a single fatty-acid side chain. Concentrations are reported in $\mu$ m. The median analytical variance of EPIC-Potsdam samples was $7.3 \%$ within-plate coefficient of variation and $11.3 \%$ between-plates coefficient of variation. ${ }^{38}$ After exclusion of metabolites below the limit of detection $(n=30)$ and those with high analytical variance in our samples $(n=6), 127$ metabolites (17 acylcarnitines, 14 AA, one hexose, 81 glycerophospholipids and 14 SM) were remaining for the present analysis.

\section{Statistical analysis}

Descriptive statistics were calculated as mean and s.d. for continuous variables and percentage for categorical variables.

As most of the metabolite concentrations were normally distributed they were not transformed. In order to conduct robust and coherent analyses of all measured metabolites nonparametric statistics were chosen. To select most body fat-relevant metabolites related with both WC (as aggregating measure of abdominal subcutaneous and visceral fat) and $\mathrm{HC}$ (as aggregating measure of gluteofemoral fat, muscles and bone structure), associations between metabolites and BMI (as marker of excess body fat and aggregating marker of body fat mass and lean mass) were first evaluated by means of Spearman's partial rank correlation coefficients $(r)$. Correlation analyses were adjusted for height (per $\mathrm{cm})$, age (per year), education (current in training/no certificate/skill; professional school; collage of higher education, university), smoking (never smoker; exsmoker; smoker), physical activity (inactive; moderately inactive; moderately active; active), alcohol consumption ( $0 \mathrm{~g}$ per day; $>0-6 \mathrm{~g}$ per day; $>6-12 \mathrm{~g}$ per day; $>12-24 \mathrm{~g}$ per day; $>24-60 \mathrm{~g}$ per day; $>60-96 \mathrm{~g}$ per day; $>96 \mathrm{~g}$ per day), fasting status (fasting, non-fasting) and prevalent hypertension and were performed stratified by sex. As correlations of 127 metabolites with BMI, WC and HC showed almost identical relations with $\mathrm{BMI}$ and $\mathrm{WC}$, but markedly lower relations with $\mathrm{HC}$ (data not presented), it appears unlikely that we missed relevant metabolites specifically related to abdominal and/or gluteofemoral body mass by the BMI-based selection process. Thereafter, metabolites with $r \geqslant 10.20 \mathrm{I}$ in men and/or women were analyzed regarding body shape. Median metabolite concentrations (95\% confidence intervals) associated with abdominal fat were assessed across sex-specific tertiles of WC additionally adjusted for HC, whereas median metabolite concentrations associated with gluteofemoral body mass were assessed across sex-specific tertiles of HC additionally adjusted for WC. These statistical models with mutual adjustment of WC and $\mathrm{HC}$ allowed the investigation of metabolites related to abdominal fat mass (approximated through differing $\mathrm{WC}$ and constant $\mathrm{HC}$ ) and gluteofemoral body mass (approximated through differing HC and constant WC). Further adjustments corresponded to the adjustment set described for the correlation analyses. The lowest tertile of metabolite concentrations were used as reference categories. Distribution-free 95\% confidence intervals were calculated as described by Hahn and Meeker. ${ }^{39}$ The margin of 10.20 I was chosen to focus the analyses on metabolites of most metabolic relevance regarding abdominal and gluteofemoral body mass. This margin was considered as sufficiently high, as above this margin clearest trends across tertiles of abdominal and gluteofemoral body mass were observed.

All statistical analyses were carried out with SAS Enterprise Guide release 6.1 (SAS Institute, Cary, NC, USA). Statistical codes available from the corresponding author.

\section{RESULTS}

The present study population comprised 2270 adults $(63.0 \%$ women) free of chronic diseases with a mean age of $49.7 \pm 8.9$ years (mean \pm s.d.). Baseline characteristics are presented in Table 1 . The study population was slightly overweight according to BMI. WC was at the upper boundaries of normal in both sexes.

Out of 127 metabolites a set of 23 metabolites showed $r \geqslant 10.20$ I with overall body mass, measured by BMI, in men and women, respectively (Table 2). This metabolite set included three AA (tyrosine, valine, isoleucine), five PCaa (C38:3, C38:4, C40:5, C40:6, C42:0), 11 PCae (C34:2, C34:3, C36:2, C38:2, C40:4, C42:3, C42:4, C42:5, C44:4, C44:5, C44:6), three lysoPC (C17:0, C18:1, C18:2) and one SM (C18:1). AA, the SM, and shorter-chained PCaa $(\leqslant C 40)$ 
Table 1. Baseline characteristics of the study population

\begin{tabular}{|c|c|c|c|}
\hline Baseline characteristics & $\begin{array}{c}\text { Total } \\
(\mathrm{n}=2270)\end{array}$ & $\begin{array}{c}\text { Men } \\
(\mathrm{n}=839)\end{array}$ & $\begin{array}{l}\text { Women } \\
(\mathrm{n}=1431)\end{array}$ \\
\hline \multicolumn{4}{|l|}{ Anthropometry } \\
\hline BMI $\left(\mathrm{kg} \mathrm{m}^{-2}\right)$ & $25.9(4.1)$ & $26.5(3.4)$ & $25.5(4.5)$ \\
\hline Waist circumference $(\mathrm{cm})$ & $85.0(12.5)$ & $93.4(9.7)$ & $80.1(11.2)$ \\
\hline Hip circumference $(\mathrm{cm})$ & $100.6(7.9)$ & $99.7(6.0)$ & $101.1(8.8)$ \\
\hline \multicolumn{4}{|l|}{ Education } \\
\hline $\begin{array}{l}\text { Current in training/no } \\
\text { certificate/skill }\end{array}$ & 36.74 & 31.35 & 39.90 \\
\hline Professional school & 24.58 & 15.38 & 29.98 \\
\hline $\begin{array}{l}\text { Collage of higher education, } \\
\text { university }\end{array}$ & 38.68 & 53.28 & 30.12 \\
\hline \multicolumn{4}{|l|}{ Smoking status } \\
\hline Never smoker & 47.62 & 29.92 & 58.00 \\
\hline Ex-smoker & 31.54 & 43.86 & 24.32 \\
\hline Smoker & 20.84 & 26.22 & 17.68 \\
\hline \multicolumn{4}{|l|}{ Physical activity } \\
\hline Inactive & 19.38 & 18.24 & 20.06 \\
\hline Moderately inactive & 39.60 & 38.26 & 40.39 \\
\hline Moderately active & 24.01 & 24.91 & 23.48 \\
\hline Active & 17.00 & 18.59 & 16.07 \\
\hline \multicolumn{4}{|l|}{ Alcohol consumption } \\
\hline $0 \mathrm{~g}$ per day & 2.78 & 2.98 & 2.66 \\
\hline$>0$ to $6 \mathrm{~g}$ per day & 38.85 & 16.33 & 52.06 \\
\hline$>6$ to $12 \mathrm{~g}$ per day & 20.97 & 15.97 & 23.90 \\
\hline$>12$ to $24 \mathrm{~g}$ per day & 18.11 & 24.79 & 14.19 \\
\hline$>24$ to $60 \mathrm{~g}$ per day & 16.34 & 32.78 & 6.71 \\
\hline$>60$ to $96 \mathrm{~g}$ per day & 2.47 & 5.84 & 0.49 \\
\hline$>96$ g per day & 0.48 & 1.31 & - \\
\hline
\end{tabular}

Continuous variables are presented as means (s.d.). Categorical variables are presented in percent. Anthropometric variables and covariates are presented in total and separated by sex. Alcohol consumption category $>96 \mathrm{~g}$ per day includes only men.

were positively correlated with $\mathrm{BMI}$, whereas PCae, lysoPCs and the longer-chained PCaa (C42) were negatively correlated. There were no differences in direction of correlations between men and women, but strength of correlations differed between sexes. Within the set of 23 metabolites, AA, PCaa, longer-chained PCae (C44) and lysoPC (C18) were stronger correlated in men, whereas the remaining PCae ( $\leqslant C 42)$, lysoPC $(C 17)$ and the SM were stronger correlated in women. Significantly higher $r$ were solely seen for tyrosine in men and PCae C38:2 in women.

The set of 23 metabolites was thereupon analyzed regarding metabolite concentrations related to body shape. For each tertile of WC-adjusted for $\mathrm{HC}$ and other variables and thus reflecting abdominal fat - and HC - adjusted for WC and other variables and thus reflecting gluteofemoral body mass-, the median concentration of the selected metabolites was calculated. Figure 1 (men) and Figure 2 (women) show the results of these calculations for each metabolite illustrating the results for WC given a constant $\mathrm{HC}$ $\left(W C_{H C}\right)$ in red and the results for $\mathrm{HC}$ given a constant WC $\left(\mathrm{HC}_{\mathrm{WC}}\right)$ in blue. For the reader's convenience the direction of the medians across tertiles were additionally displayed by arrows below the graphics of the metabolite results. To gain a comprehensive view, correlations with BMI as well whether metabolites had been already identified as being associated with pre-diabetes and/or type-2-diabetes in previous studies are additionally indicated. We found that median metabolite concentrations across tertiles of $W C_{H C}$ often show a similar pattern as the correlation of the metabolites with BMI. This similarity was most pronounced for
Table 2. Spearman's partial rank correlation coefficients $r(95 \% \mathrm{Cl})$ of serum metabolites with BMI, stratified by sex

\begin{tabular}{|c|c|c|}
\hline Metabolites & $\begin{array}{c}\text { Men } \\
(\mathrm{n}=839)\end{array}$ & $\begin{array}{c}\text { Women } \\
(\mathrm{n}=1431)\end{array}$ \\
\hline \multicolumn{3}{|l|}{ Amino Acids } \\
\hline Tyrosine* & $0.29(0.22 ; 0.35)$ & $0.12(0.07 ; 0.17)$ \\
\hline Valine & $0.24(0.17 ; 0.30)$ & $0.20(0.15 ; 0.25)$ \\
\hline Isoleucine & $0.22(0.15 ; 0.28)$ & $0.17(0.12 ; 0.22)$ \\
\hline \multicolumn{3}{|c|}{ Diacyl-phosphatidylcholines } \\
\hline PCaa C $38: 3^{*}$ & $0.34(0.27 ; 0.39)$ & $0.25(0.20 ; 0.30)$ \\
\hline PCaa C38:4 & $0.24(0.17 ; 0.30)$ & $0.13(0.08 ; 0.18)$ \\
\hline PCaa C40:5 & $0.20(0.13 ; 0.27)$ & $0.08(0.02 ; 0.13)$ \\
\hline PCaa C40:6 & $0.23(0.16 ; 0.29)$ & $0.11(0.06 ; 0.16)$ \\
\hline PCaa C42:0 & $-0.24(-0.30 ;-0.17)$ & $-0.19(-0.24 ;-0.14)$ \\
\hline \multicolumn{3}{|c|}{ Acyl-alkyl-phosphatidylcholines } \\
\hline PCae C34:2 & $-0.13(-0.20 ;-0.06)$ & $-0.21(-0.26 ;-0.16)$ \\
\hline PCae C34:3* & $-0.21(-0.27 ;-0.14)$ & $-0.27(-0.31 ;-0.22)$ \\
\hline PCae C36:2* & $-0.17(-0.23 ;-0.10)$ & $-0.27(-0.32 ;-0.22)$ \\
\hline PCae C38:2* & $-0.13(-0.20 ;-0.06)$ & $-0.26(-0.31 ;-0.22)$ \\
\hline PCae C40:4 & $-0.14(-0.21 ;-0.07)$ & $-0.21(-0.26 ;-0.16)$ \\
\hline PCae C42:3 & $-0.18(-0.25 ;-0.11)$ & $-0.22(-0.27 ;-0.17)$ \\
\hline PCae C42:4* & $-\mathbf{0 . 2 4}(-0.30 ;-0.17)$ & $-0.26(-0.30 ;-0.21)$ \\
\hline PCae C42:5 & $-0.22(-0.28 ;-0.15)$ & $-0.21(-0.26 ;-0.16)$ \\
\hline PCae C44:4 & $-0.22(-0.28 ;-0.15)$ & $-0.18(-0.23 ;-0.13)$ \\
\hline PCae C44:5 & $-0.20(-0.27 ;-0.13)$ & $-0.16(-0.21 ;-0.10)$ \\
\hline PCae C44:6 & $-0.22(-0.28 ;-0.15)$ & $-0.20(-0.25 ;-0.15)$ \\
\hline \multicolumn{3}{|c|}{ Lyso-phosphatidylcholines } \\
\hline lysoPC a C17:0 & $-0.16(-0.23 ;-0.10)$ & $-0.21(-0.26 ;-0.16)$ \\
\hline lysoPC a C18:1 & $-0.24(-0.30 ;-0.17)$ & $-0.17(-0.22 ;-0.12)$ \\
\hline lysoPC a C18:2* & $-0.25(-0.31 ;-0.19)$ & $-0.20(-0.25 ;-0.15)$ \\
\hline \multicolumn{3}{|l|}{ Sphingomyelins } \\
\hline SM C18:1 & $0.13(0.06 ; 0.20)$ & $0.21(0.16 ; 0.26)$ \\
\hline
\end{tabular}

Abbreviations: a, acyl; aa, diacyl; ae, acyl-alkyl; BMl, body mass index; $\mathrm{Cl}$, confidence interval; PC, phosphatidylcholine; SM, sphingomyelin. Correlation coefficients are adjusted for body height, age, education, smoking, physical activity, alcohol consumption, fasting status and prevalent hypertension, and rounded to two decimal places. Correlation coefficients $\geqslant 10.20 \mathrm{I}$ are marked in bold. Metabolites with correlation coefficients $\geqslant 10.25 \mathrm{I}$ in men and/or women are additionally marked by asterisks (*). Fatty-acid side chains were abbreviated $C x: y$ where $x$ represents the number of carbon atoms and $y$ the number of double bounds.

metabolites with $r \geqslant 10.20$ I such as tyrosine, valine, isoleucine, PCaa C38:3, C38:4, C40:5, C40:6, PCae C42:4, C44:4, lysoPC C18:1 and C18:2 in men and valine, PCaa C38:3, PCae C34:2, C34:3, C36:2, C38:2, C40:4, C42:3, C42:4, C42:5, C44:6 and lysoPC C18:2 in women. Metabolites with $r \geqslant 10.20 \mathrm{I}$ and no clear trend across tertiles of $W C_{H C}$ such as PCaa C42:0, PCae C34:3, C42:5, C44:5 and C44:6 in men and lysoPC C17 in women showed at least tendencies of concordant directions of associations, whereas metabolites with $r<10.20$ I predominantly showed not more than tendencies across the tertiles. In contrast, median metabolite concentrations across tertiles of $\mathrm{HC}_{\mathrm{WC}}$ were less concordant with the results of $\mathrm{BMI}$ and $\mathrm{WC}_{\mathrm{HC}}$. This is of particular interest as associations with the analyzed metabolites were not studied before. Some metabolites showed median metabolite concentrations across tertiles of $\mathrm{HC}_{\mathrm{WC}}$ in the same direction as across tertiles of $\mathrm{WC}_{\mathrm{HC}}$, such as tyrosine, PCaa C38:3, C38:4, C40:5, C40:6, lysoPC C17:0, C18:1, C18:2 and SM C18:1 in men and tyrosine, PCaa C38:3, C38:4, lysoPC C18:1, C18:2 and SM C18:1 in women. However, some metabolites showed opposite directions as across tertiles of $\mathrm{WC}_{\mathrm{HC}}$, such as isoleucine, PCaa C42:0, and PCae C34:3, C42:3, C42:4, C42:5, C44:4, C44:6 in men and valine, isoleucine, PCaa 


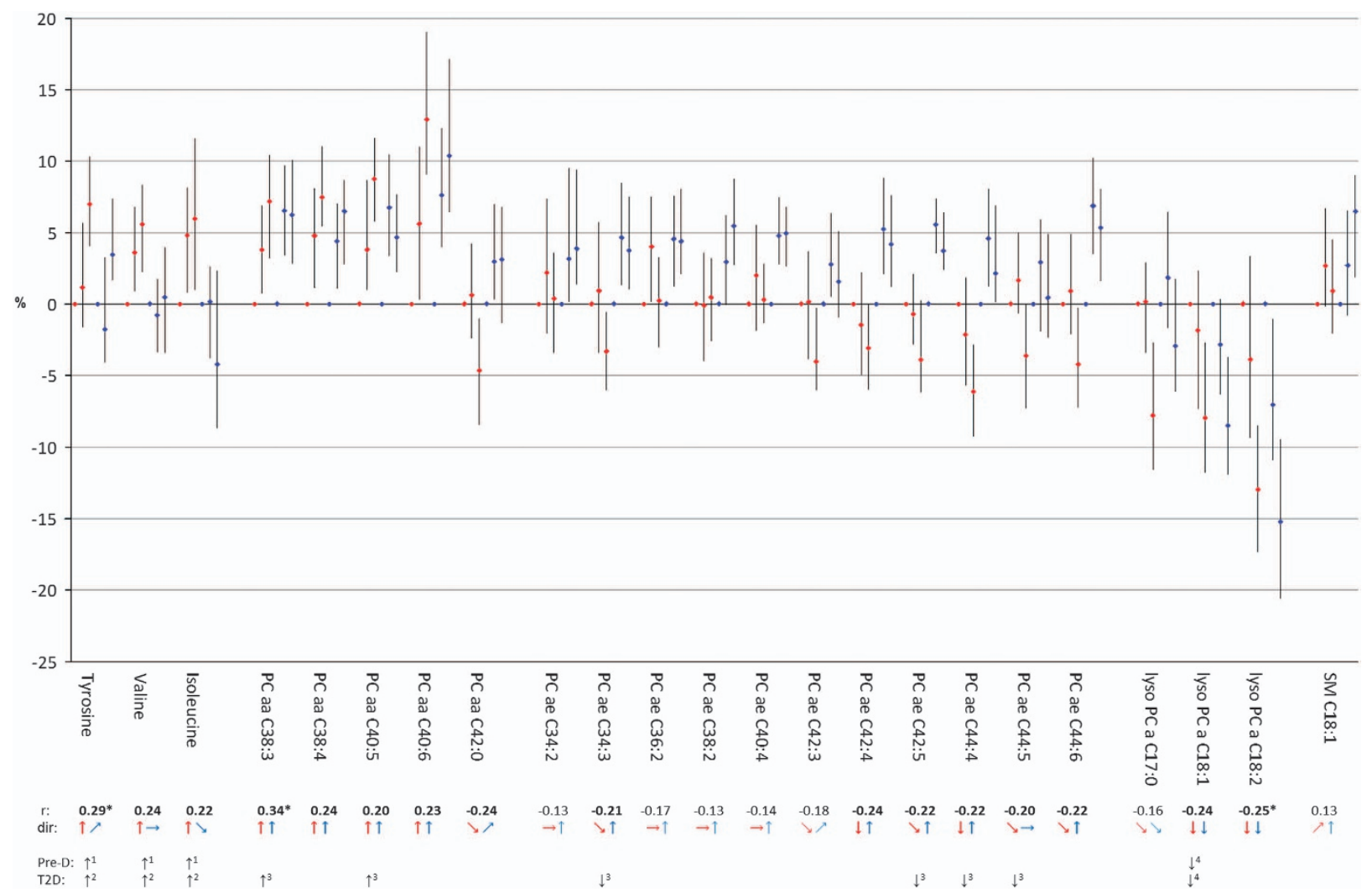

Figure 1. Median serum concentrations across tertiles of WC (red) and HC (blue) in relation to corresponding reference groups in men. Reference groups are lowest tertiles of WC and HC, respectively. Tertile classifications are as follows: WC tertile $1: W C<89 \mathrm{~cm}, W C$ tertile 2 : $89 \mathrm{~cm} \leqslant W C<97 \mathrm{~cm}$, WC tertile 3: WC $\geqslant 97 \mathrm{~cm}$; HC tertile 1: HC $<97 \mathrm{~cm}$, HC tertile 2: $97 \mathrm{~cm} \leqslant \mathrm{HC}<102 \mathrm{~cm}, \mathrm{HC}$ tertile $3: \mathrm{HC} \geqslant 102 \mathrm{~cm}$. Adjustment sets included the following covariates: body height, age, education, smoking, physical activity, alcohol consumption, fasting status, prevalent hypertension as well as $\mathrm{HC}$ for concentrations across tertiles of WC and WC for concentrations across tertiles of HC, respectively. To gain a comprehensive view, correlation coefficients $(r)$ with BMI are added below: $r \geqslant 10.20$ I are marked in bold, $r \geqslant 10.25 \mathrm{I}$ are additionally marked by asterisk. Directions (dir) of median serum concentrations across tertiles are presented by arrows: $\uparrow$ or $\downarrow=$ clear trend; $\nearrow$ or $\searrow=$ tendency; $\rightarrow=$ marginal tendency. Metabolites already associated with pre-diabetes (Pre-D) and/or type-2-diabetes (T2D) in previous studies are additionally marked: $1=$ Würtz et al.; ${ }^{45} 2$ Wang et al.; ${ }^{46} 3=$ Floegel et al. ${ }^{24} 4=$ Wang-Sattler et al. ${ }^{21} \mathrm{HC}$, hip circumference; WC, waist circumference.

C40:5, C40:6, C42:0, PCae C34:2, C34:3, C40:4, C42:4, C42:5, C44:4, C44:5 and C44:6 in women. Moreover, some metabolites showed clear trends across tertiles of $\mathrm{HC}_{\mathrm{WC}}$, but not more than tendencies across tertiles of $W C_{H C}$, such as PCae C34:2, C36:2, C38:2, C40:4 in men; and some metabolites showed clear trends across tertiles of $\mathrm{WC}_{\mathrm{HC}}$, but not more than tendencies across tertiles of $\mathrm{HC}_{\mathrm{WC}}$, such as valine and PCae C44:5 in men and PCae C36:2, C38:2, C42:3 and lysoPC C17:0 in women. Consequently, the 23 metabolites could be subdivided into four groups: those being related in the same way with both measurements of body shape-the gluteofemoral body mass and the abdominal fat mass, those being inversely related with the measurements of body shape, and those being specifically related with either the gluteofemoral body mass or the abdominal fat mass. We noted that the assignment of the metabolites to one of the four groups is also sex-specific.

\section{DISCUSSION}

We analyzed 23 serum metabolites related to body mass with $r \geqslant 10.20 \mathrm{I}$ at least in one sex for their relations with body mass either located at the waist or at the hip. We observed that a body mass deposition at the hip has unique and sometimes opposite relations with metabolites compared with a deposition at the waist. The deposition at the waist shows similar relations with metabolites than overall fatness (BMI) and includes metabolites known to be related to type-2-diabetes.

First, we like to discuss the type of metabolites being considered as reflecting overall fatness that also includes specific deposition particularly at the waist. We identified two branchedchain (valine, isoleucine) and one aromatic (tyrosine) AA related to overall fatness. These results are in line with previous findings suggesting that both branched-chain (BCAA) and aromatic AA were positively associated with excess body weight. ${ }^{16,40}$ Moreover, the observed stronger correlations in men basically support the hypothesis that males have higher rates of protein turnover than females. ${ }^{16}$ We could further show that shorter-chained PCaa $(\leqslant \mathrm{C} 40)$ were positively correlated with overall fatness, and longerchained PCaa (C42) and all PCae and lysoPC were negatively correlated. Thereby, PCaa were stronger related in men, whereas PCae were stronger related in women. In agreement with our findings lower PCae ${ }^{41,42}$ and lower lysoPC concentrations were observed in obese individuals in other studies. ${ }^{15,17}$ However, concerning lysoPC evidence is inconsistent as Pietiläinen et al. ${ }^{41}$ reported higher lysoPC concentrations related to obesity. The present study also identified one SM (C18:1) related to overall body mass, conforming to previous results. ${ }^{43}$

The strong similarity of findings regarding metabolites associated with $\mathrm{BMI}$ and $\mathrm{WC}_{\mathrm{HC}}$ is not surprising as $\mathrm{BMI}$ and $\mathrm{WC}$ are 


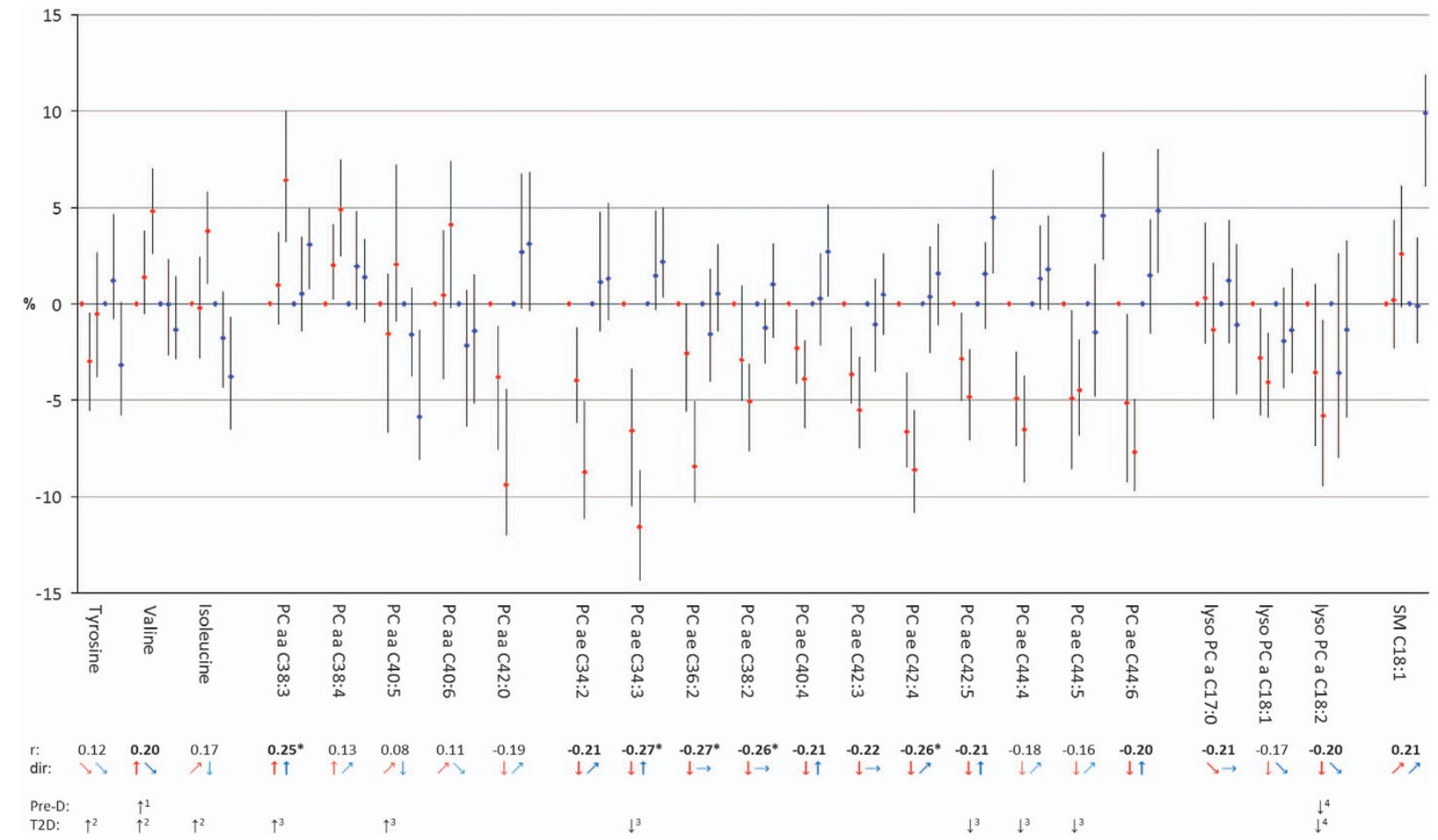

Figure 2. Median serum concentrations across tertiles of WC (red) and HC (blue) in relation to corresponding reference groups in women. Reference groups are lowest tertiles of WC and $\mathrm{HC}$, respectively. Tertile classifications are as follows: WC tertile $1:$ WC $<74 \mathrm{~cm}, \mathrm{WC}$ tertile 2 : $74 \mathrm{~cm} \leqslant W C<83.5 \mathrm{~cm}$, WC tertile 3: $\geqslant 83.5 \mathrm{~cm} ; \mathrm{HC}$ tertile $1: \mathrm{HC}<97 \mathrm{~cm}, \mathrm{HC}$ tertile 2: $97 \mathrm{~cm} \leqslant \mathrm{HC}<103.5 \mathrm{~cm}, \mathrm{HC}$ tertile $3: \mathrm{HC} \geqslant 103.5 \mathrm{~cm}$. Adjustment sets included the following covariates: body height, age, education, smoking, physical activity, alcohol consumption, fasting status, prevalent hypertension as well as HC for concentrations across tertiles of WC and WC for concentrations across tertiles of HC, respectively. To gain a comprehensive view, correlation coefficients $(r)$ with BMI are added below: $r \geqslant 10.20 \mathrm{I}$ are marked in bold, $r \geqslant 10.25 \mathrm{I}$ are additionally marked by asterisk. Directions (dir) of median serum concentrations across tertiles are presented by arrows: $\uparrow$ or $\downarrow=$ clear trend; $\nearrow$ or $\searrow=$ tendency; $\rightarrow=$ marginal tendency. Metabolites already associated with pre-diabetes (Pre-D) and/or type-2-diabetes (T2D) in previous studies are additionally marked: $1=$ Würtz et al.; ${ }^{45} 2=$ Wang et al.; ${ }^{46} 3=$ Floegel et al.; ${ }^{24} 4=$ Wang-Sattler et al. ${ }^{21} \mathrm{HC}$, hip circumference; WC, waist circumference.

highly correlated. This confirms a strong positive correlation between the underlying metabolite networks of BMI and WC $(r=0.99)$ within the same data set. ${ }^{44}$ This finding is also supporting our decision to select metabolites for further analysis based on correlations with $\mathrm{BMI}$ and on the margin of $10.20 \mathrm{I}$. Above this margin, clearest trends of median metabolite concentrations across tertiles of abdominal and gluteofemoral body mass were observed. Metabolites specifically related to abdominal fat were observed for different metabolites in men (valine, PCae C44:5) and women (PCae C36:2 C38:2, C42:3, lysoPC C17:0). Interestingly, the two metabolites observed in men were previously identified as risk metabolites of pre-diabetes ${ }^{45}$ and type-2-diabetes. ${ }^{24,46}$ They might act as early indicators of adverse health implications specifically related to abdominal fat. The exact mechanism that explains elevated health risks associated with WC is not firmly established. It is assumed that visceral fat has an important etiological role of diabetes by release of free fatty acids directly from visceral fat into hepatic circulation, resulting in insulin resistance, hyperinsulinemia, ${ }^{27,28}$ increased gluconeogenesis and dyslipidemia. ${ }^{47,48}$ So far, little attention has been given to associations between type of body shape and metabolite profiles in metabolically healthy individuals. The revealed metabolites are possibly involved in underlying mechanisms resulting in such disorders. Increased protein catabolism secondary to insulin resistance has been suggested as possible explanation for increased BCAA in obesity. ${ }^{49}$ In moderate upper body obesity increased proteolysis and impairment of insulin's antiproteolytic action was found. ${ }^{50,51}$ Furthermore, tissue-specific alterations in
BCAA metabolism, in the liver and adipose tissue but not in muscle, may contribute to increased plasma concentrations of BCAA in obesity. ${ }^{49}$ Besides higher serum concentrations of BCAA we found lower serum concentration of PCae and lysoPC to be related with increased abdominal fat. Plasma phospholipids, mostly secreted by the liver, are abundantly present in all classes of lipoproteins. PCae were found to be inversely correlated with plasma triglycerides. ${ }^{24}$ Furthermore, higher levels of PCae were observed to be related with improved insulin sensitivity and reduced insulin secretion; ${ }^{24}$ in addition, they may act antioxidative and prevent lipoprotein oxidation. ${ }^{42}$

The novel insight relates to the body mass at the hip-mostly reflecting subcutaneous fat, muscle mass and bone structure (pelvic width). It seems as if increased body mass at this site has its own metabolic consequences, probably with less-adverse implications than large overall fat volumes and abdominal fat when the metabolites related to this deposition were taken into consideration. The interpretation of metabolic consequences related to body mass at the hip may vary between sexes. Although gluteofemoral fat and pelvic width may be the main components of $\mathrm{HC}$ in women, pelvic width and muscles may be the main components in men. On the one hand, underlying mechanisms of less-adverse implications may be related to subcutaneous fat, which has lower levels of basal lipolysis and lipolytic stimulation compared with visceral fat, potentially resulting in lower flux of free fatty acids into the blood. ${ }^{47}$ Furthermore, it has been suggested that subcutaneous fat may act as a sink for circulating free fatty acids. ${ }^{48}$ Subcutaneous fat is additionally supposed to 
consist of smaller adipocytes that have relatively higher insulin sensitivity. ${ }^{52}$ Owing to differences in body fat distribution metabolic impact of subcutaneous fat may vary between men and women. On the other hand, less-adverse implications may be related to muscle mass, the main target organ for insulin and one of the sites of insulin resistance. ${ }^{30} \mathrm{~A}$ lower $\mathrm{HC}$ possibly indicates relatively small gluteal and leg muscle mass. This could be more apparent in men owing to lower gluteofemoral fat storage compared with women. It has been shown in healthy men that relatively small leg muscle mass is related to an elevated waist-tohip ratio ${ }^{53}$ and to different glucose levels between Indian and Swedish men. ${ }^{54}$

We could note that also deposition of body mass at this site show for some metabolites similar relations with overall and abdominal fat. Those metabolites showing corresponding associations in men and women included tyrosine, PCaa C38:3, C38:4, lysoPC C18:1, C18:2, and SM C18:1—with mostly clearer trends in men. We also observed some metabolites related in men only (PCaa C40:5, C40:6, lysoPC C17:0). Tyrosine, PCaa C38:3, C40:5 and lysoPC C18:2 were already found to be related to pre-diabetes and/or type-2-diabetes. ${ }^{21,24,45,46}$ Metabolites with concordant relations with abdominal and gluteofemoral body mass might be considered as reflecting large body volumes and include indicators of elevated metabolic risks associated with excess body weight in general wherever it is located. Higher levels of aromatic AA have been related to excess body fat, insulin ${ }^{55}$ and insulin resistance. ${ }^{12}$ Choline-containing phospholipids such as PCaa and lysoPC represent the major components of cellular membranes. ${ }^{56}$ Their blood concentration may be influenced by hepatic de novo synthesis and redistribution from plasma membranes. ${ }^{57}$ As constituents of lipoproteins, PCaa have been related to hepatic secretion of triyglceride-rich VLDL particles and HDL. ${ }^{56}$ The observed associations of sphingomyelin SM C18:1 with higher abdominal and gluteofemoral body mass seem to be attributed to both fat and muscle mass, what is in line with previous studies. ${ }^{58,59}$

However, we also observed metabolites showing opposite associations regarding type of body shape. In men and women, isoleucine, PCaa C42:0, PCae C34:3, C42:4, C42:5, C44:4 and C44:6 exhibited this property-with mostly clearer trends in women. We also observed some metabolites related in women only (valine, PCaa C40:5, C40:6, PCae C34:2, C40:4, C44:5). Valine, isoleucine, PCaa C40:5, PCae C34:3, C42:5, C44:4 and C44:5 were already found to be related to pre-diabetes and/or type-2-diabetes. ${ }^{24,45,46}$ To our surprise, the direction of relations with the compartment 'waist' corresponded to the findings with pre-diabetes and/or type-2-diabetes, and the relations with the compartment 'hip' were opposite. This finding strengthens the view that gluteofemoral body mass deposition might even induce metabolic processes protecting against type-2-diabetes. In women, fat deposition at the hip region and regional differences in adipocyte metabolism (lipoprotein lipase activity and lipolysis) are more pronounced than in men. ${ }^{60}$ In the present study a possible protective role of lager hips was found to be more apparent in women than in men. This finding suggests that metabolic processes protecting against type-2-diabetes might be particularly linked to subcutaneous fat deposition.

Metabolites specifically related to gluteofemoral body mass only were solely observed in men (PCae C34:2, C36:2, C38:2, C40:4) and comprised none of the identified risk metabolites for prediabetes and/or type-2-diabetes. The present findings suggest that gluteofemoral body mass deposition has different metabolic consequences and support the hypothesis that increased gluteofemoral body mass presumably goes along with lessadverse health implications.

Among the strengths of the present study are the use of standardized procedures for the assessment of anthropometry by trained personnel and the availability of concentrations of metabolites with known identity comprising different classes. Determination by the validated AbsoluteIDQ p150 kit allowed simultaneous quantification using a modern high-throughput technique, which has been approved and standardized. Further strengths of our study are the possibility to consider relevant confounding variables and the exclusion of individuals with chronic diseases to avoid effects of accompanying metabolic changes. The limitations of the study refer to its cross-sectional nature and the use of anthropometrical measurements, which are only rough estimations of the true body composition. Measurements of body composition obtained by direct methods such as dual-energy X-ray absorptiometry, magnetic resonance imaging or bodyscanner accurately and precisely quantify compartments of body composition. Such methods require special and expensive equipment and are often unsuitable to screen large samples. Indubitably, mutual adjustment of WC and HC can only provide an approximation of metabolite concentration differences related to abdominal and gluteofemoral body mass, respectively. Nevertheless, to receive hints for differences in associations related to abdominal and gluteofemoral body mass, it seems methodologically appropriate. A further limitation relates to the selection of metabolites, which is highly artificial and of rather small extent when considering what's technically possible in metabolomics. Furthermore, variances of metabolite concentrations depend not only on biological variability, but also on the precision of the measurements, which might have influenced our assumption of metabolic relevance. Based on findings of a reliability study, metabolites below the detection limit and those with high analytical variance were excluded to ensure valid measurements. ${ }^{38}$

In conclusion, the study confirmed several metabolite associations with BMI as anthropometric marker of overall body mass, provided metabolic profiles concerning $W C_{H C}$ as marker of abdominal fat mass and revealed specific metabolic profiles for $\mathrm{HC}_{\text {WC }}$ as marker of gluteofemoral body mass differing from those of overall body mass and abdominal fat. Thereby, we observed sex-specific differences. Metabolites already identified as being associated with pre-diabetes and/or type-2-diabetes were assignable to the three abdominal fat-related metabolite groups and appear independent from their associations with gluteofemoral body mass, underscoring the lower adverse metabolic consequences by this compartment. The observation of independent associations of metabolites with gluteofemoral body mass as well as opposite relations with abdominal and gluteofemoral body mass even suggest that increased deposition in the gluteofemoral area could possibly reduce the risk of type-2-diabetes owing to beneficial metabolic impacts. Future studies using direct measurements of body composition need to further validate these findings.

\section{CONFLICT OF INTEREST}

The authors declare no conflict of interest.

\section{ACKNOWLEDGEMENTS}

The study was supported by a grant from the Federal Ministry of Education and Research, Germany (Bundesministerium für Bildung und Forschung) to the Nationales Genomforschungsnetz NGFN DE (Funding number: 01GS0821) and the Competence Network on Obesity (Kompetenznetz Adipositas; Funding number: 01GI1121B).

\section{REFERENCES}

1 World Health Organization. Global Status Report on Noncommunicable Diseases 2010. World Health Organization: Geneva, 2011.

2 Stevens J, Truesdale KP. Obesity: fat distribution. In: Caballero B, Allen L, Prentice A (eds). Encyclopedia of Human Nutrition, 2nd edn. Elsevier Ltd: Oxford, UK, 2005, pp 392-399. 
3 Després JP, Moorjani S, Lupien PJ, Tremblay A, Nadeau A, Bouchard C. Regional distribution of body fat, plasma lipoproteins, and cardiovascular disease. Arteriosclerosis 1990; 10: 497-511.

4 Fox CS, Massaro JM, Hoffmann U, Pou KM, Maurovich-Horvat P, Liu CY et al. Abdominal visceral and subcutaneous adipose tissue compartments: association with metabolic risk factors in the Framingham Heart Study. Circulation 2007; 116: 39-48.

5 Manolopoulos KN, Karpe F, Frayn KN. Gluteofemoral body fat as a determinant of metabolic health. Int J Obes 2010; 34: 949-959.

6 Buemann B, Astrup A, Pedersen O, Black E, Holst C, Toubro S et al. Possible role of adiponectin and insulin sensitivity in mediating the favorable effects of lowerbody fat mass on blood lipids. J Clin Endocrinol Metab 2006; 91: 1698-1704.

7 Benn RT. Some mathematical properties of weight-for-height indices used as measures of adiposity. Br J Prev Soc Med 1971; 25: 42-50.

8 Han TS, McNeill G, Seidell JC, Lean ME. Predicting intra-abdominal fatness from anthropometric measures: the influence of stature. Int J Obes Relat Metab Disord 1997; 21: 587-593.

9 Rankinen T, Kim SY, Pérusse L, Després JP, Bouchard C. The prediction of abdominal visceral fat level from body composition and anthropometry: ROC analysis. Int J Obes Relat Metab Disord 1999; 23: 801-809.

10 Neamat-Allah J, Wald D, Hüsing A, Teucher B, Wendt A, Delorme $S$ et al. Validation of anthropometric indices of adiposity against whole-body magnetic resonance imaging--a study within the German European Prospective Investigation into Cancer and Nutrition (EPIC) cohorts. PLoS One 2014; 9: e91586.

11 Oberbach $A$, Blüher $M$, Wirth $H$, Till $H$, Kovacs $P$, Kullnick $Y$ et al. Combined proteomic and metabolomic profiling of serum reveals association of the complement system with obesity and identifies novel markers of body fat mass changes. J Proteome Res 2011; 10: 4769-4788.

12 Newgard CB, An J, Bain JR, Muehlbauer MJ, Stevens RD, Lien L et al. A branched-chain amino acid-related metabolic signature that differentiates obese and lean humans and contributes to insulin resistance. Cell Metab 2009; 9: 311-326.

13 Fiehn O, Garvey WT, Newman JW, Lok KH, Hoppel CL, Adams SH. Plasma metabolomic profiles reflective of glucose homeostasis in non-diabetic and type 2 diabetic obese African-American women. PLoS One 2010; 5: e15234.

14 Huffman KM, Shah SH, Stevens RD, Bain JR, Muehlbauer M, Slentz CA et al. Relationships between circulating metabolic intermediates and insulin action in overweight to obese, inactive men and women. Diabetes Care 2009; 32: 1678-1683.

15 Kim JY, Park JY, Kim OY, Ham BM, Kim HJ, Kwon DY et al. Metabolic profiling of plasma in overweight/obese and lean men using performance liquid chromatography and Q-TOF mass spectrometry (UPLC-Q-TOF MS). J Proteome Res 2010; 9: 4368-4375.

16 Morris C, O'Grada C, Ryan M, Roche HM, Gibney MJ, Gibney ER et al. The relationship between BMI and metabolomic profiles: a focus on amino acids. Proc Nutr Soc 2012; 71: 634-638.

17 Heimerl S, Fischer M, Baessler A, Liebisch G, Sigruener A, Wallner S et al. Alterations of plasma lysophosphatidylcholine species in obesity and weight loss. PLoS One 2014; 9: e111348.

18 Martin FP, Montoliu I, Collino S, Scherer M, Guy P, Tavazzi I et al. Topographical body fat distribution links to amino acid and lipid metabolism in healthy obese women. PLoS One 2013; 8: e73445.

19 Szymańska E, Bouwman J, Strassburg K, Vervoort J, Kangas AJ, Soininen P et al. Gender-dependent associations of metabolite profiles and body fat distribution in a healthy population with central obesity: towards metabolomics diagnostics. OMICS 2012; 16: 652-667.

20 Newgard CB. Interplay between lipids and branched-chain amino acids in development of insulin resistance. Cell Metab 2012; 15: 606-614.

21 Wang-Sattler R, Yu Z, Herder C, Messias AC, Floegel A, He Y et al. Novel biomarkers for pre-diabetes identified by metabolomics. Mol Syst Biol 2012; 8: 615.

22 Zhang X, Wang Y, Hao F, Zhou X, Han X, Tang H et al. Human serum metabonomic analysis reveals progression axes for glucose intolerance and insulin resistance statuses. J Proteome Res 2009; 8: 5188-5195.

23 Larsen PJ, Tennagels N. On ceramides, other sphingolipids and impaired glucose homeostasis. Mol Metab 2014; 3: 252-260.

24 Floegel A, Stefan N, Yu Z, Mühlenbruch K, Drogan D, Joost HG et al. Identification of serum metabolite associated with risk of type 2 diabetes using a targeted metabolomic approach. Diabetes 2013; 62: 639-648.

25 Suhre K, Meisinger C, Döring A, Altmaier E, Belcredi P, Gieger C et al. Metabolic footprint of diabetes: a multiplatform metabolomics study in an epidemiological setting. PLoS One 2010; 5: e13953.

26 Barber MN, Risis S, Yang C, Meikle PJ, Staples M, Febbraio MA et al. Plasma lysophosphatidylcholine levels are reduced in obesity and type 2 diabetes. PLoS One 2012; 7: e41456.
27 Després JP, Lemieux S, Lamarche B, Prud'homme D, Moorjani S, Brun LD et al. The insulin resistance-dyslipidemic syndrome: contribution of visceral obesity and therapeutic implications. Int J Obes Relat Metab Disord 1995; 19(suppl): S76-S86.

28 Randle PJ. Regulatory interactions between lipids and carbohydrates: the glucose fatty acid cycle after 35 years. Diabetes Metab Rev 1998; 14: 263-283.

29 Snijder MB, Dekker JM, Visser M, Bouter LM, Stehouwer CD, Yudkin JS et al. Trunk fat and leg fat have independent and opposite associations with fasting and postload glucose levels: the Hoorn study. Diabetes Care 2004; 27: 372-377.

30 Seidell JC, Han TS, Feskens EJ, Lean ME. Narrow hips and broad waist circumferences independently contribute to increased risk of non-insulindependent diabetes mellitus. J Intern Med 1997; 242: 401-406.

31 Boeing H, Korfmann A, Bergmann MM. Recruitment procedures of EPIC-Germany. European Investigation into Cancer and Nutrition. Ann Nutr Metab 1999; 43: 205-215.

32 Kroke A, Bergmann MM, Lotze G, Jeckel A, Klipstein-Grobusch K, Boeing H. Measures of quality control in the German component of the EPIC study. European Prospective Investigation into Cancer and Nutrition. Ann Nutr Metab 1999; 43: $216-224$.

33 Klipstein-Grobusch K, Georg T, Boeing H. Interviewer variability in anthropometric measurements and estimates of body composition. Int J Epidemiol 1997; 26(Suppl 1): S174-S180.

34 Boeing H, Wahrendorf J, Becker N. EPIC-Germany -- A source for studies into diet and risk of chronic diseases. European Investigation into Cancer and Nutrition. Ann Nutr Metab 1999; 43: 195-204.

35 Haftenberger M, Lahmann PH, Panico S, Gonzalez CA, Seidell JC, Boeing $\mathrm{H}$ et al. Overweight, obesity and fat distribution in 50- to 64-year-old participants in the European Prospective Investigation into Cancer and Nutrition (EPIC). Public Health Nutr 2002; 5: 1147-1162.

36 Floegel A, von Ruesten A, Drogan D, Schulze MB, Prehn C, Adamski J et al. Variation of serum metabolites related to habitual diet: a targeted metabolomic approach in EPIC-Potsdam. Eur J Clin Nut 2013; 67: 1100-1108.

37 Römisch-Margl W, Prehn C, Bogumil R, Röring C, Suhre K, Adamski J. Procedure for tissue sample preparation and metabolite extraction for high-throughput targeted metabolomics. Metabolomics 2012; 8: 133-142.

38 Floegel A, Drogan D, Wang-Sattler R, Prehn C, Illig T, Adamski J et al. Reliability of serum metabolite concentrations over a 4-month period using a targeted metabolomic approach. PLoS One 2011; 6: e21103.

39 Hahn GJ, Meeker WQ (eds). Statistical Intervals: a Guide for Practitioners. John Wiley \& Sons, Inc.: Hoboken, NJ, USA, 1991.

40 Xie B, Waters MJ, Schirra HJ. Investigating potential mechanisms of obesity by metabolomics. J Biomed Biotechnol 2012; 2012: 805683.

41 Pietiläinen KH, Sysi-Aho M, Rissanen A, Seppänen-Laakso T, Yki-Järvinen H, Kaprio J et al. Acquired obesity is associated with changes in the serum lipidomic profile independent of genetic effects - a monozygotic twin study. PLoS One 2007; 2: e218.

42 Wallner S, Schmitz G. Plasmalogens the neglected regulatory and scavenging lipid species. Chem Phys Lipids 2011; 164: 573-589.

43 Hanamatsu H, Ohnishi S, Sakai S, Yuyama K, Mitsutake S, Takeda H et al. Altered levels of serum sphingomyelin and ceramide containing distinct acyl chains in young obese adults. Nutr Diabetes 2014; 4: e141.

44 Floegel A, Wientzek A, Bachlechner U, Jacobs S, Drogan D, Prehn C et al. Linking diet, physical activity, cardiorespiratory fitness and obesity to serum metabolite networks: findings from a population-based study. Int J Obes (Lond) 2014; 38: 1388-1396.

45 Würtz $\mathrm{P}$, Soininen $\mathrm{P}$, Kangas $\mathrm{AJ}$, Rönnemaa $\mathrm{T}$, Lehtimäki $\mathrm{T}$, Kähönen $\mathrm{M}$ et al Branched-chain and aromatic amino acids are predictors of insulin resistance in young adults. Diabetes Care 2013; 36: 648-655.

46 Wang TJ, Larson MG, Vasan RS, Cheng S, Rhee EP, McCabe E et al. Metabolite profiles and the risk of developing diabetes. Nat Med 2011; 17: 448-453.

47 Björntorp P. "Portal" adipose tissue as a generator of risk factors for cardiovascular disease and diabetes. Arteriosclerosis 1990; 10: 493-496.

48 Frayn KN. Adipose tissue as a buffer for daily lipid flux. Diabetologia 2002; 45 1201-1210.

49 She P, Van Horn C, Reid T, Hutson SM, Cooney RN, Lynch CJ. Obesity-related elevations in plasma leucine are associated with alterations in enzymes involved in branched-chain amino acid metabolism. Am J Physiol Endocrinol Metab 2007; 293: E1552-E1563.

50 Jensen MD, Haymond MW. Protein metabolism in obesity: effects of body fat distribution and hyperinulinemia on leucine turnover. Am J Clin Nutr 1991; 53: 172-176.

51 Luzi L, Castellino P, DeFronzo RA. Insulin and hyperaminoacidemia regulate by a different mechanism leucine turnover and oxidation in obesity. Am J Physiol Endocrinol Metab 1996; 270: E273-E281.

52 Rebuffé-Scrive M, Lönnroth P, Mårin P, Wesslau C, Björntorp P, Smith U. Regional adipose tissue metabolism in men and postmenopausal women. Int J Obes 1987; 11: 347-355. 
53 Seidell JC, Björntorp P, Sjöström L, Sannerstedt R, Krotkiewski M, Kvist H. Regional distribution of muscle and fat mass in men--new insight into the risk of abdominal obesity using computed tomography. Int J Obes 1989; 13: 289-303.

54 Chowdhury B, Lantz H, Sjostrom L. Computed tomography-determined body composition in relation to cardiovascular risk factors in Indian and matched Swedish males. Metabolism 1996; 45: 634-644.

55 Felig P, Marliss E, Cahill GF Jr. Plasma amino acid levels and insulin secretion in obesity. N Engl J Med 1969; 281: 811-816.

56 Cole LK, Vance JE, Vance DE. Phosphatidylcholine biosynthesis and lipoprotein metabolism. Biochim Biophys Acta 2012; 182: 754-761.

57 Zhao X, Peter A, Fritsche J, Elcnerova $M$, Fritsche A, Häring $\mathrm{HU}$ et al. Changes of the plasma metabolome during an oral glucose tolerance test: is there more than glucose to look at? Am J Physiol Endocrinol Metab 2009; 296: E384-E393.

58 Błachnio-Zabielska A, Zabielski P, Baranowski M, Gorski J. Aerobic training in rats increases skeletal muscle sphingomyelinase and serine palmitoyltransferase activity, while decreasing ceramidase activity. Lipids 2011; 46: 229-238.
59 Straczkowski M, Kowalska I, Nikolajuk A, Dzienis-Straczkowska S, Kinalska I, Baranowski $M$ et al. Relationship between insulin sensitivity and sphingomyelin signaling pathway in human skeletal muscle. Diabetes 2004; 53: 1215-1221.

60 Rebuffé-Scrive M, Enk L, Crona N, Lönnroth P, Abrahamsson L, Smith U et al. Fat cell metabolism in different regions in women. Effect of menstrual cycle, pregnancy, and lactation. J Clin Invest 1985; 75: 1973-1976.

(i) This work is licensed under a Creative Commons Attribution 4.0 International License. The images or other third party material in this article are included in the article's Creative Commons license, unless indicated otherwise in the credit line; if the material is not included under the Creative Commons license, users will need to obtain permission from the license holder to reproduce the material. To view a copy of this license, visit http://creativecommons.org/licenses/ by/4.0/

(c) The Author(s) 2016 